\title{
Man-machine interface in a submarine command and weapon control system: Features and design experience $\dagger$
}

\author{
JOHAN H. AAS, KARSTEN BRÅTHEN, \\ ERIK NORDØ‡ and OLE Ø. ØRPEN§
}

\begin{abstract}
Keywords: Man-machine systems, human factors, underseas systems, prototyping, system analysis, guidance systems, target tracking.

Important man-machine interface (MMI) issues concerning a submarine command and weapon control system (CWCS) such as crew organization, automation level and decision support are discussed in this paper. Generic submarine CWCS functions and operating conditions are outlined. Detailed, dynamic and real-time prototypes were used to support the MMI design. The prototypes are described and experience with detailed prototyping is discussed. Some of the main interaction principles are summarized and a restricted example of the resulting design is given. Our design experience and current work have been used to outline future perspectives of MMI design in naval CWCSs. The need for both formal and experimental approaches is emphasized.
\end{abstract}

\section{Introduction}

Operations in a subsurface environment are characterized by the limited availability of information on surrounding activities. A submarine observes the world through different sensors, where sonars are the most important. Although contemporary submarines are equipped with sensors producing increasing amounts of data, the acoustical propagation, the ambient noise and the steady reduction in vessel noise compound the observation problem. In addition to data reduction and information extraction from observing the surroundings, the submarine crew face complex tactical problems such as making priorities and decisions in an uncertain multi-target situation. The total workload calls for a high degree of automation, which further requires extensive modeling of the environment. Automatic functions may prove satisfactory under normal conditions, but due to the stochastic, nonlinear and time-variant sound propagation in the ocean, the crew must have a set of interactive tools available.

Facing the development of the next generation CWCS for conventional submarines with its high performance requirements and partly incomplete and unclear function descriptions, we recognized a need for a prototyping activity. The work was commissioned by the Royal Norwegian Navy and has involved close cooperation with submarine officers. The study has been focused on:

The task allocation and the coordination between operators.

Received August 1988.

$\dagger$ This paper was presented at the 3rd IFAC/IFIP/IEA/IFORS Conference on ManMachine Systems; Analysis, Design and Evaluation, June 14-16, 1988, Oulu, Finland and is reprinted with permission of IFAC.

$\ddagger$ Norwegian Defence Research Establishment, Division for Electronics, N-2007 Kjeller, Norway.

$\S \mathrm{A} / \mathrm{S}$ Geoteam, 0311 Oslo 3, Norway. 
The automation level.

Interaction principles.

The detailed design of displays and operator interaction.

This paper describes some of the features of a submarine CWCS which are pertinent to the MMI. Most of the characteristics of submarine CWCSs are of a generic nature and are common to other types of CWCSs, and civil surveillance and command systems operating under time constraints. The paper addresses the prototyping, and outlines some of the results and lessons learned. The last section deals with trends in specification and design of these kind of systems.

\section{Submarine CWCS overview}

A submarine CWCS is a real-time data collection, data processing, weapon control and decision support system. The CWCS gathers data from the sensors, communication links and the operators on the activity of surface vessels and submarines in the environment. The CWCS then processes the gathered data to generate tactical support information, fire control and weapon guidance data. The functions of the CWCS for the engagement of one target are initiated sequentially and run concurrently until the end of the engagement. The main functions of one engagement are shown in the composition graph (Alford 1985) in Fig. 1 and can be summarized as follows:

Contact acquisition involves gathering measurements from established and new contacts. Multiple tracks from different sensor systems are then correlated to resolve which tracks originate from the same vessel (contact association).

Classification is a process to distinguish friendly units from enemy units and to classify the vessel.

Target Motion Analysis (TMA) involves the estimation of the target position, speed and course. An important part of TMA is algorithms for the processing of sonar bearings.

The threat analysis involves assessing the threat posed by enemy units to ones own submarine.

The engagement analysis involves the prediction of the probabilities of success for different weapon engagement alternatives.

Weapon engagement comprises preparing the weapon, executing the launch and guiding the weapon.

A commanding officer makes important decisions based on his mission, the result of classification, TMA, threat and engagement analysis where priorities between the different targets are taken into account.

\section{Main characteristics of the CWCS MMI}

What implications for the MMI can be derived from the fundamental characteristics of the CWCS and the way the submarine is operated?

\subsection{Crew organization}

The CWCS is manned with a varying number of operators depending on the tactical situation at hand. It should be possible for one person to operate the CWCS 


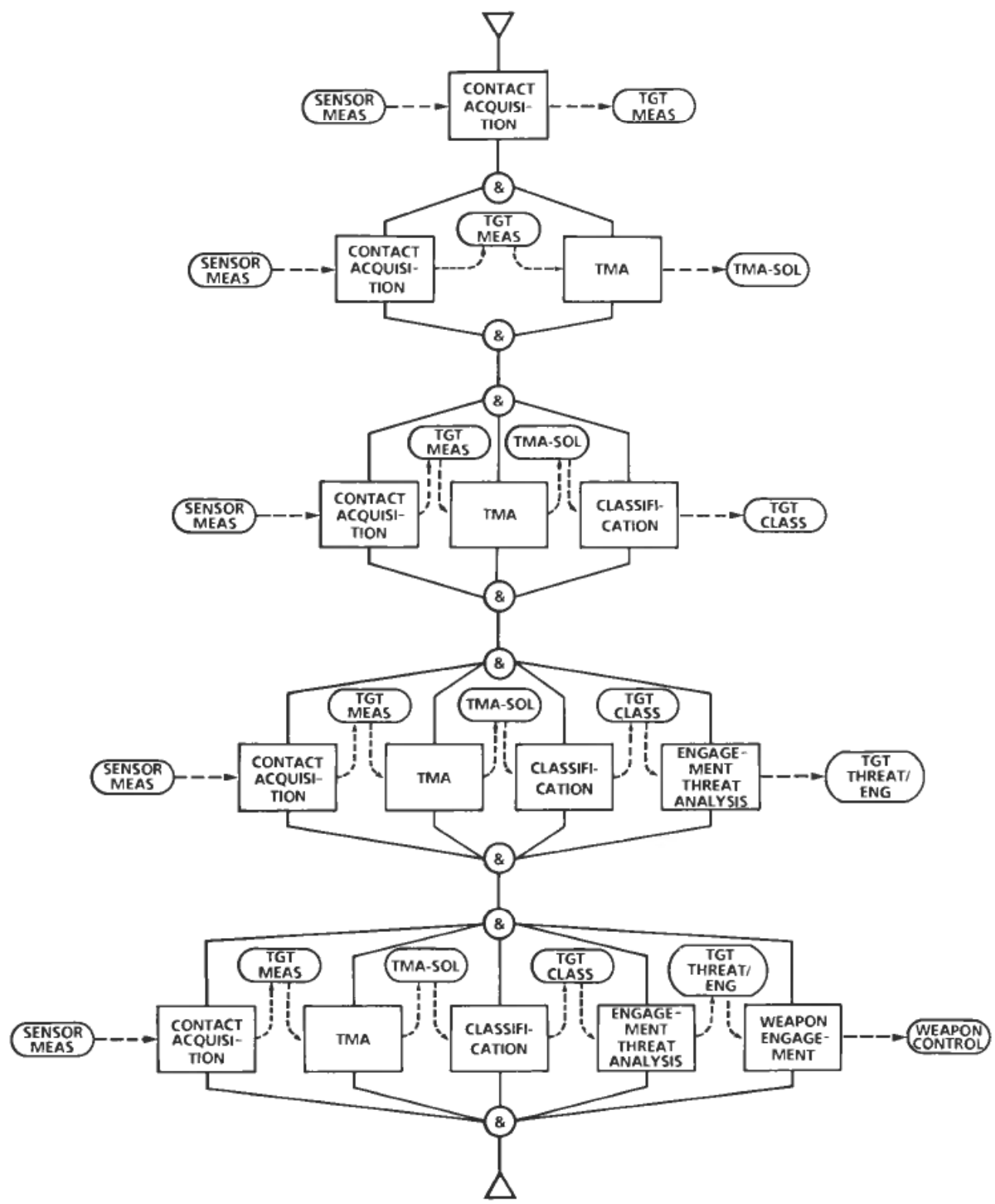

Figure 1. CWCS functional sequence.

in a patrol situation. In an alert situation, several operators should be allowed to share the total workload on multi-purpose consoles with the following modes:

A tactical mode for contact association, classification, threat analysis, allocation of tasks to other operators and approval of results for tactical use.

A TMA mode for monitoring and improvement of TMA solutions.

A weapon mode for engagement analysis and weapon engagement.

There are a number of weapon and sensor operators in addition to the CWCS operators. The crew are hierarchically organized with the commanding officer as the 
task coordinator and tactical decision maker. The hierarchy is maintained under all operating conditions. To achieve the necessary flexibility in the number of operators, the CWCS must be able to provide all functions and information on one console. With multiple consoles, there must be provisions for information transfer and coordination between the operators and the commanding officer. There must be features that prohibit important decisions from being made by officers other than the commanding officer.

\subsection{Automation level}

Several variables influence the degree of operator interaction:

The number of targets in the area.

The available time and information.

The amount of formalized information in the CWCS.

The experience of the operator.

The operator stress.

The large variation in the variables from a multi-target engagement to a patrol situation, makes a fixed automation level unacceptable. We want to reduce the operator workload while still enabling the operators to influence the solutions by exploiting information that is not formalized. To allow for this flexibility, only a small subset of the tasks needs operator support. These tasks are mandatory checkpoints throughout the engagement and operator decisions are needed to proceed, e.g. target allocation and launch of weapon. The other tasks are designed for a varying degree of operator interaction. Automatic algorithms always produce solutions which are utilized if no operator interaction has been performed. In addition to the automatic algorithms, a repertoire of support functions is available to assist the operator when a more interactive procedure is feasible and required. These functions rely to a varying degree on the formalized information in the system and the operator's information processing and decision-making. Thus, a hierarchical operator interaction approach is achieved within each function, from a completely automatic procedure to manual control where the CWCS only acts as a bookkeeper. The model-basis for the automatic procedures is an added justification for this hierarchical operator interaction. Typically, the models are stochastic, non-linear and time-variant. Even though the algorithms to a certain degree are adaptive, more interactive support tools must be available to achieve robustness under adverse operating conditions.

Bearing only TMA is characterized by its low observability and non-linear algorithms (Borstad 1983). Thus, the effective use of all measurements as well as additional information is of vital importance. Additional information could be a range constraint from the acoustical propagation model. Integrating additional operator information into the stochastic models (e.g. the non-linear estimators of the TMA), forces the operator to formalize his subjective information statistically. This fusion of information has proved to have many deficiencies. Consistent weighting of the subjective information and the measurements, and finding a straightforward way for the operator to formalize his knowledge to the CWCS are two such deficiencies. Our approach has been to add methods relying on geometric representation. These methods enable the operator to test hypotheses on a trial-and-error basis until the 
resulting motion state complies with his additional information and the sensor measurement. For an overview of TMA in the ocean environment see e.g. Hassab (1983).

\subsection{Decision support}

The CWCS has a number of different ways to support the operator in his decision making. Some of the prevailing characteristics of these tools can be outlined as follows:

Dynamic simulation models to predict consequences of different actions and engagement approaches. For example, the system is able to simulate a weapon engagement. The simulation includes the dynamics of the submarine, the target and the weapon.

Acoustical propagation models to assist in the threat and engagement analysis. Such as models to estimate the detection ranges of targets, own submarine and weapons.

Non-linear estimators enabling the operator to select measurements and express additional information without restriction in the time domain.

Database query systems to classify and identify targets.

Operational rules of thumb applied to the formalized situation assessment model to suggest solutions and courses of actions. For example, determine a complete weapon engagement.

Auditory information from the sonar systems to supplement the synthetic situation presentation on the screens.

The operator is able to test different assumptions and hypotheses using a trial and error approach, assumptions and hypotheses. The models are optimized to operate in the real-time dynamic decision-making situation.

\subsection{Console hardware}

The CWCS consoles are multi-purpose consoles, i.e. the hardware is identical, but they can be configured to the different console modes. The hardware for the CWCS multi-purpose consoles was basically designed before the MMI prototyping was started. The console is shown in Fig. 2. The main interaction devices are:

Two high resolution colour graphic raster scan screens.

A programmable touch-sensitive plasma panel.

An alphanumeric keyboard.

A trackball with associated control keys.

\section{Prototyping}

The benefits of prototyping the MMI are well-established, see e.g. Alavi (1984). Prototyping is especially useful in order to establish, maintain and validate the user requirements. Additionally, existing MMI design guidelines are usually insufficient to derive the MMI for a specific application. A prototype is also important as a common model among the various engineering teams involved. 


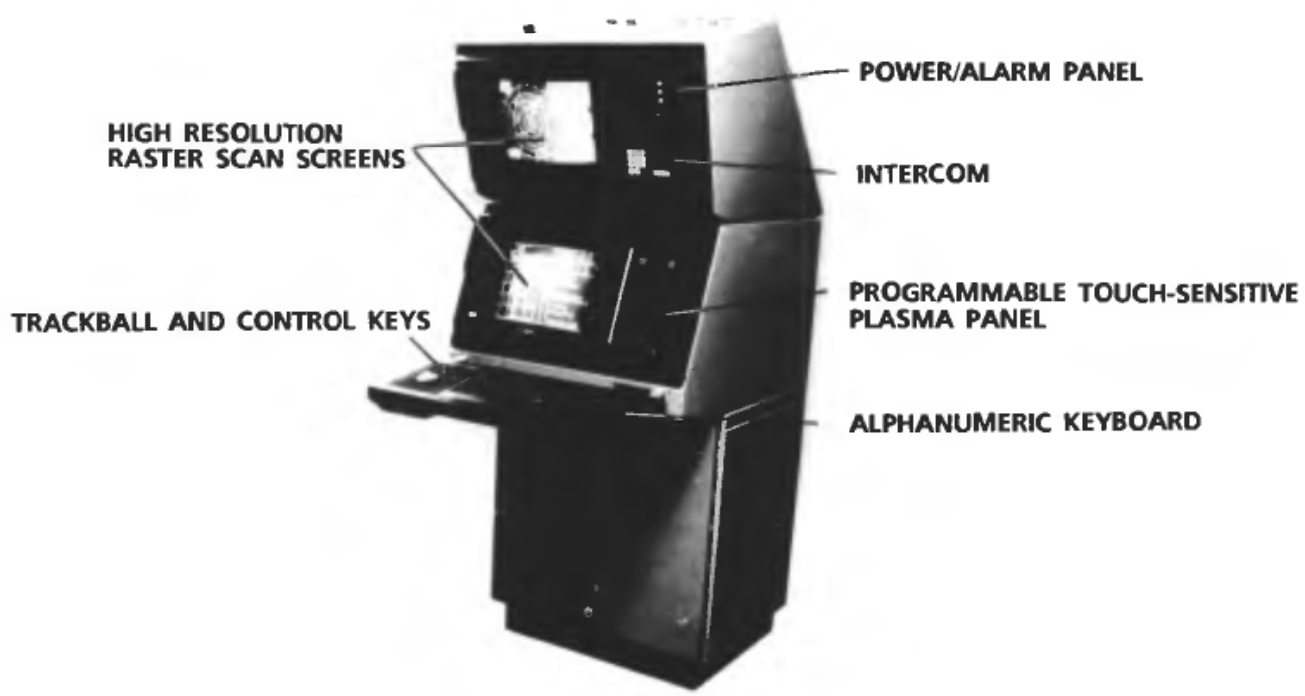

Figure 2. CWCS console.

The requirements for many of the CWCS functions were unclear and incomplete. Some of the functions were performed by the operator without computer support in earlier systems. The integration of such functions into the system represented an innovative approach that required experimentation. The results of the automatic algorithms provide important contributions to the situation assessment. Therefore, it is important to consider the dynamics and uncertainty of these results when the MMI is developed. This means that it is necessary to simulate the environment and the automatic algorithms in detail. The main purpose of the prototypes was to test the TMA and the weapon functions in a representative real-time environment. Our ambition was not to perform rigorous experiments, but to gain enough feedback during different kinds of evaluations to have confidence in the proposed MMI. Important aspects of these evaluations were:

Decomposition and sequencing of tasks.

Choice of interaction techniques.

Detailed information presentation.

Time and performance studies.

The design of the console and the selection of interaction devices were not part of our work.

The prototypes utilized interaction devices which were identical to those of the CWCS, but different computers (DEC VAX-11/750 and RAMTEK 9460). The prototype development resulted in a real-time system of 60000 lines of source code. The detailed simulation of the environment and the automatic algorithms amount to approximately one third of this software.

The prototypes were frequently used by ourselves, the Navy and engineers who implemented the CWCS. Apart from a lot of informal demonstrations, a number of more systematic and complete evaluations were performed. Relatively simple but realistic tactical situations were used in these evaluations. The situations evolved in real-time and the prototypes were used ad hoc. A total of 9 submarine officers with 
different degrees of experience at sea (from 3 months to 6 years) participated in 1 to 3 major evaluation periods each. The programme included about 1 day of training and a total of 6 hours of testing. The tests were recorded by video and observers took notes. After the tests, the officers completed a comprehensive questionnaire with about 200 questions. An interview was then performed to extract more personal and detailed comments.

\section{CWCS user interaction concepts}

The conceptual model of user interaction is basically command-driven. The interaction style is object-oriented, giving the operator access to objects (e.g. targets and weapons) by direct manipulation (Shneiderman 1983). To avoid selection of a large number of different plots, we organized the interaction around a small number of plots which are divided into several windows with the possibility of exchanging the information content in each window. The tiled windows are only reconfigurable within strict limitations.

Each of the console modes operates basically on two main plots, a situation overview plot and a so-called workplot. Thus, the three workplots are the tactical, the TMA and the weapon workplot. The two main plots for each console mode define an information hierarchy. The situation overview plot condenses all the formalized situation assessment in the system. The workplot is restricted to a single object and gives access to the complete information base for that object.

The plasma panel is used for function selection. A set of main functions with associated functions is defined for each plot. In addition to this function tree, an additional tree is defined for plot-related functions (e.g. scale and information suppression/selection). The physical layout of the two function trees on the plasma panel is shown in Fig. 3. The initial dialogue had a tight coupling between windows and plasma panel function activation. However, tests on the prototype showed that a more independent operation was much less error-prone.

\section{Example of resulting design}

The TMA can basically be handled automatically, but robustness and performance requirements generate a set of functions which requires operator interaction. The following outlines a typical sequence of an operator dialogue.

The situation overview plot and the TMA workplot are displayed on the upper and lower screen respectively. The TMA plot is illustrated in Fig. 4 and divided into the following windows:
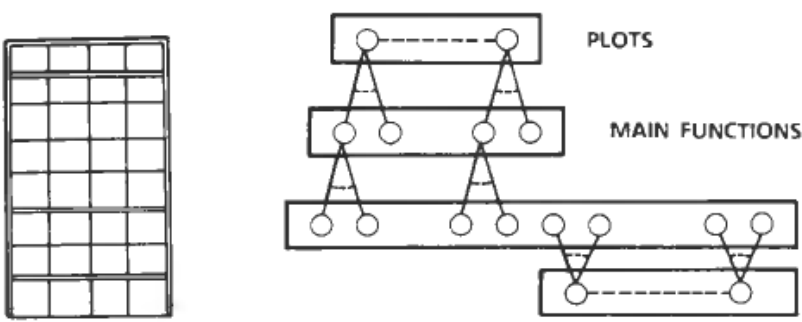

ASSOCIATED FUNCTIONS

PLOT RELATED FUNCTIONS

Figure 3. Plasma panel layout. 


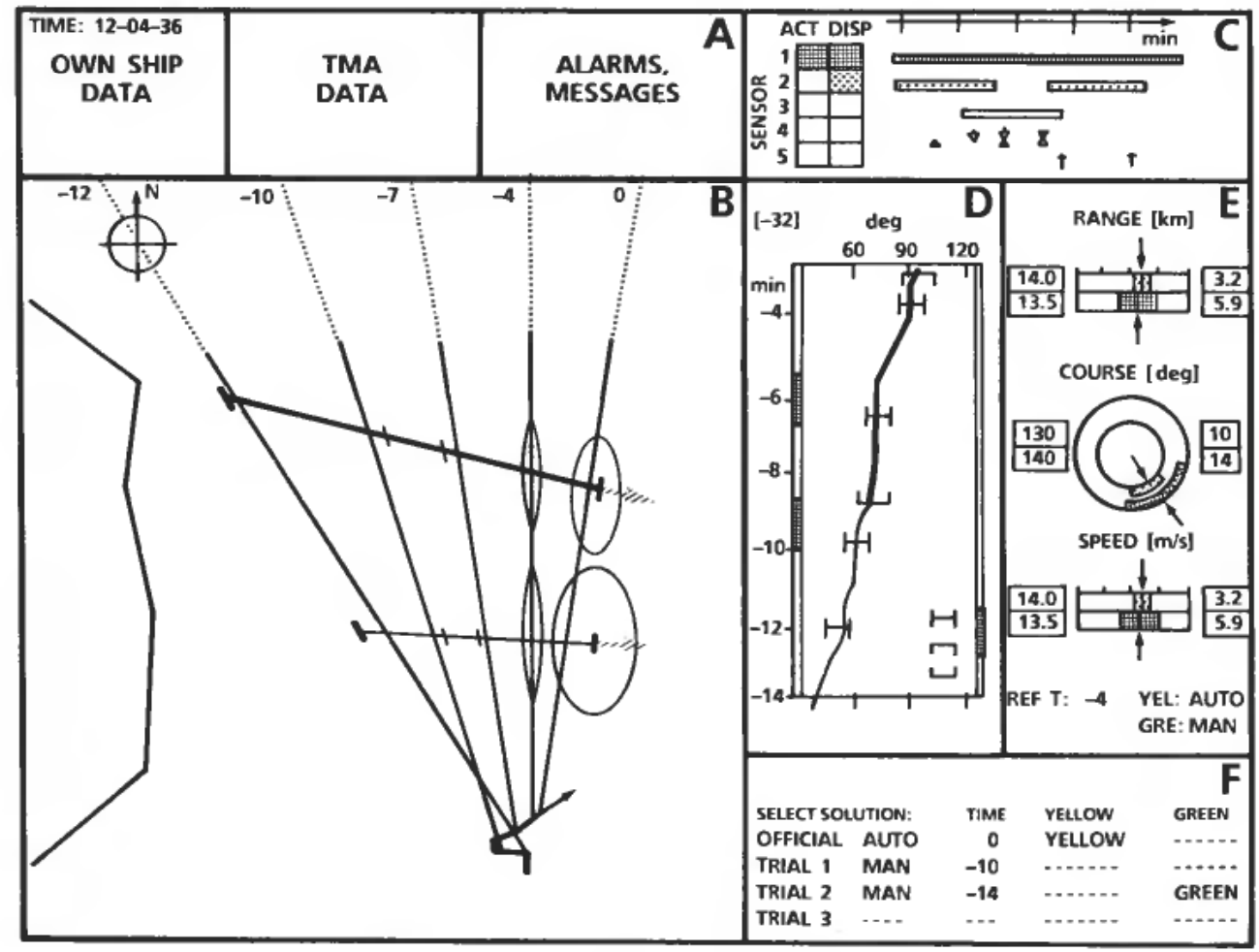

Figure 4. Layout of the TMA workplot.

(A) Status window. Displaying the status information, warnings and alarms.

(B) Geographic window. Contains shore lines, submarine movements, geometric presentation of selected sensor measurements and two estimated target trajectories corresponding to different TMA solutions.

(C) Sensor overview window. Displaying the data- acquisition status of the sensors.

(D) Sensor measurement window. Displaying the measurements from one or more sensors as a function of time.

(E) Solution window. Displaying the two TMA solutions.

(F) Menus and forms window.

Assume that the TMA operator is ordered by the commanding officer to evaluate and, if possible, improve a TMA solution for an important target. The operator selects the target on the situation overview plot by using the trackball and switches the interaction to the TMA workplot. The operator inspects the acquisition of sensor measurements and the convergence of the automatic TMA solution. Based on available measurements and some additional information the operator decides to generate an alternative solution denoted as a 'trial-solution'. In order to make a trial-solution, the operator selects a specific sensor in the sensor overview window with the trackball. Sequences and single measurements are then selected in the sensor measurement window also with the trackball. The selected measurements are automatically displayed in the geographic window. The operator enters his addi- 
tional information consisting of a speed estimate and a range constraint as a trialsolution in the solution window by using the keyboard. He decides to use his trial-solution as prior information to be used together with the selected measurements in a TMA algorithm (e.g. an extended Kalman filter). The processing is started by selecting the algorithm on the plasma panel. The operator evaluates the resulting trial-solution geometrically in the geographic window. He decides that the additional information was not weighted correctly relative to his confidence in the measurements and modifies the solution.

\section{Design experience and future perspectives}

When MMI is specified, many different views need to be described. Natural language and graphics, for instance showing the screen layout and sequences, were used in our dialogue specifications. Though this approach provided a flexible, rich description, our experience is that it becomes too ambiguous and incomplete for large systems. Additionally, no support is given to preserve consistency throughout the system. The operator tasks, the MMI and the machine functions should be developed and described as integral parts to take full account of the potential of a more formal specification and description method. However, the most widely-used system development methods do not clearly visualize the MMI (Floyd 1986). In our experience the main requirements for a system development method which takes equal account of the operator tasks, the MMI and the machine function are first of all that the starting point of the description should be the scenarios. Thus, event sequences for describing the system behaviour over time is a dimension of utmost importance. Static models, such as Structured Analysis and Design Technique (Ross 1977), do not have the ability to express this view. Concurrency should also be included to allow the development of an integrated model of the operator tasks and the machine. The information flow, which is a key concept for most contemporary system development methods, is equally important for the description of manmachine systems. Another typical characteristic of the existing methods is their strict hierarchical structure. Allocating functionality to different operators and subsystems, from an integrated man-machine system description, generates a network of hierarchies.

To our knowledge no system development method applicable to large-scale naval CWCSs is available today. Nevertheless, approaches from distributed computer system design (Alford 1985) offer favourable characteristics and can possibly be augmented to include the unique features of the human 'subsystem'. In other words, the interaction techniques by which information is transferred from the human to the computer and the coding of the information to the human.

The experimental support gained by the prototypes was valuable in the MMI design. A lot of important details of the proposed MMI were changed as a result of the evaluations. However, the main concepts pvoved to be sound. The most important ways of getting feedback from the evaluations were to observe the officers during the tests and to receive their informal comments. The use of the video-tapes was too time-consuming to be of any significant value to the design process. The importance of creating realistic and realtime situations to obtain valid results should be emphasized. The implementation of the prototypes contributed to a more consistent and complete MMI proposal. During the long development-time the prototypes served as hands-on models of the MMI to the end-users, thereby reducing 
uncertainty. The prototypes were also useful as a means of transferring the MMI specification to the implementation team.

MMI design with extensive use of prototypes is an iterative process. A large effort was expended to develop the prototypes and perform the necessary changes between the major evaluations. Apart from typical maintenance problems in software-systems, the difficulties with obtaining a stable system that allowed ad-hoc operation by naive users were the most prevalent. In our experience, it is important to stress the quality requirements of the prototype software. Computer-assisted tools should be utilized as a means of trying different alternatives and responding effectively to end-user feedback. In recent years the so-called User Interaction Management Systems (UIMS) have been developed. These are helpful in implementing a prototype to support the conceptual design of the MMI. Dynamic systems such as a submarine CWCS are characterized by real-time constraints and ultimate relations between the machine algorithms and the MMI. Therefore, detailed prototypes simulating vital parts of the system must support the system development. The detailed dynamic prototypes must include real-time aspects and real algorithms to be supportive both for the MMI and the algorithm design. For these prototypes, today's UIMS have a less profound effect on the prototyping effort. Reusable simulation components of the different physical processes and algorithms play an equally important role.

\section{Conclusion}

The first system was installed last year. The building of a CWCS has introduced many difficult MMI design problems. The various operating conditions required a flexible allocation of tasks between the machine and the operator. The integration of automatic and human decision-making, given uncertain and incomplete information, was especially challenging. The design has changed from function activation by dedicated panels on earlier systems, to contemporary screen-based interaction techniques. During the development, which spanned approximately 4 years, the prototypes proved valuable as a common reference to the Navy's procurement office, to the end-users, to the software implementation team at the contractor and to our development team. The importance of prototypes as 'physical specifications' for information and knowledge transfer between the development teams has perhaps not been fully recognized in the literature. Detailed, dynamic and real-time prototypes which include processing algorithms should be utilized to support the MMI design in many systems. Hitherto, UIMS have had limited value for this kind of prototyping.

The MMI was described in natural language and with graphics. We learned that more formal descriptions were needed. However, we have not found description methods which incorporate the operator tasks, the MMI and the machine functions into an integrated model, which is applicable to the development of large dynamic systems. Important concepts of such models are: event and time sequences, function/task concurrency and performance, information flow and network of function/task hierarchies.

The experimental and formal approaches will both have widespread effects on how we develop software-intensive dynamic systems, such as naval CWCSs, in which the human plays a significant role. The two approaches, the subjective, hands-on and physical model and the formal, objective, hierarchical and top-down 
document-based model, complement each other. This requires an augmentation of development methods for software systems to include important aspects of the MMI. Tools to support detailed, dynamic and real-time prototypes are also needed. Much promising work along these lines has been done (Kloster and Zellweger 1987), and is currently being further developed. To evaluate such approaches a development framework of real and complex applications must be used.

\section{REFERENCES}

AlAVI, M. (1984). An assessment of the prototyping approach to information systems development. Communications of the ACM, 27, 556-563.

ALFORD, M. (1985). SREM at the age of eight; the distributed design system. IEEE Computer, $18,36-46$.

BorstaD, A. J. (1983). Bearing only target motion analysis estimation characteristics. Proceedings First IASTED Applied Control and Identification Symposium, Copenhagen, Denmark, 5-25, 5-31.

FLoYD, C. (1986). A comparative evaluation of system development methods. In T. W. Olle, H. G. Sol and A. A. Verrijn-Stuart (Eds), Information systems design methodologies: Improving the practice. (Elsevier Science Publishers B.V.) pp. 19-54.

HASSAB, J. C. (1983). Contact localization and motion analysis in an ocean environment: A perspective. IEEE Journal of Oceanic Engineering, 8, 136-147.

Kloster, G. V. and ZelLweGer, A. (1987). Engineering the man-machine interface for airtraffic control. IEEE Computer, 20, 47-62.

Ross, D. T. (1977). Structured Analysis (SA): A language for communicating ideas. IEEE Transactions on Software Engineering, 3, 16-34.

ShNeIDERMAN, B. (1983). Direct manipulation: A step beyond programming languages. IEEE Computer, 16, 57-69. 